\title{
and its Applications
}

\section{The rise and fall of L-spaces, II}

\author{
Sehie Park ${ }^{a, b}$ \\ a The National Academy of Sciences, Republic of Korea, Seoul 06579; and \\ ${ }^{b}$ Department of Mathematical Sciences, Seoul National University, Seoul 08826, KOREA
}

\begin{abstract}
In 2005, Ben-El-Mechaiekh, Chebbi, and Florenzano obtained a generalization of Ky Fan's 1984 KKM theorem on the intersection of a family of closed sets on non-compact convex sets in a topological vector space. They also extended the Fan-Browder fixed point theorem to multimaps on non-compact convex sets. Since then several groups of the L-space theorists introduced coercivity families and applied them to L-spaces, H-spaces, etc. In this article, we show that better forms of such works can be deduced from a general KKM theorem on abstract convex spaces in our previous works. Consequently, all of known KKM theoretic results on L-spaces related coercivity families are extended to corresponding better forms on abstract convex spaces.

This article is a continuation of our [38] and a revised and extended version of [34].
\end{abstract}

Keywords: KKM theorem, Fan's 1961 KKM lemma, 1984 KKM theorem, Fan-Browder fixed point theorem, minimax inequality, abstract convex space, (partial) KKM space

2010 MSC: 47H04, 47H10, 49J27, 49J35, 49J53, 52A01, 54H25, 55M20, 90C47, 91A13.

\section{Introduction}

The KKM theory, first named by the author in 1992, was originally devoted to convex subsets of topological vector spaces mainly by Ky Fan and Granas, and later to the so-called convex spaces by Lassonde, to $c$-spaces (or H-spaces) by Horvath and others, to generalized convex (G-convex) spaces mainly by the present author. Since 2006, we proposed new concepts of abstract convex spaces and (partial) KKM spaces which are proper generalizations of G-convex spaces and adequate to establish the KKM theory. Consequently we have obtained a large number of new results in such frame.

Recall that a milestone on the history of the KKM theory was erected by Fan in 1961 [13]. His 1961 KKM Lemma (or the Fan-KKM theorem or the KKMF principle [5]) extended the KKM theorem to arbitrary

Email address: park35@snu.ac.kr; sehiepark@gmail.com; webpage: parksehie.com (Sehie Park) 
topological vector spaces and was applied to various problems in his subsequent papers. Moreover, his lemma was extended in 1979 and 1984 [14,15] to his $1984 \mathrm{KKM}$ theorem with a new coercivity (or compactness) condition for noncompact convex sets; see $[21,25]$.

In 1993, we introduced generalized convex (G-convex) spaces $(E, D ; \Gamma)[39]$ and, in 1998 , we derived new concept of them removing the original monotonicity restriction on them; see [21,25]. Motivated our original G-convex spaces in 1993, Ben-El-Mechaiekh, Chebbi, Florenzano, and Llinares [3,4] in 1998 introduced Lspaces $(E, \Gamma)$ and claimed incorrectly that G-convex spaces are particular to their L-spaces. Since then a number of authors followed the misconception of [4] and published incorrect obsolete articles even after we established the KKM theory on abstract convex spaces in 2006-2010.

In 2005, Ben-El-Mechaiekh, Chebbi, and Florenzano [5] obtained a generalization of Ky Fan's 1984 KKM theorem [15] on the intersection of a family of closed sets on non-compact convex sets in a topological vector space. They also extended the Fan-Browder fixed point theorem to multimaps on non-compact convex sets. This type of studies also followed by Chebbi [7,9] and others. In 2011, Chebbi, Gourdel, and Hammami [11] introduced a generalized coercivity type condition for multimaps defined on topological spaces endowed with a generalized convex structure and extended Fan's KKM theorem.

In our previous work [34] in 2013, we showed that better forms of theorems in $[5,7,8,9,11]$ can be deduced from a KKM theorem on abstract convex spaces in our sense [23-27]. Moreover, in our recent work [38], we showed that our KKM theory on abstract convex spaces improves and extends all of typical results on L-spaces. Since such studies are beyond of L-spaces, we cordially claimed that now is the proper time to give up the useless study on L-spaces and their variants FC-spaces.

After we have published [34], we found similar articles by the L-space theorists such as Chebbi [8], Hammami [17], Gourdel-Hammami [16], Chebbi [10] and Altwaijry-Ounaies-Chebbi [1] in the chronological order. These authors introduce several coercing families and applied them to corresponding KKM theorems, Fan's matching theorems, Fan-Browder fixed point theorems, minimax inequalities, etc. However, these seem to be of little use since their terminology and coercivity conditions are not practical.

In our previous work [38] in 2020, which will be called Part I, we recalled the history of relation between G-convex spaces and L-spaces, and showed that other authors' main works on L-spaces are consequences of our KKM theory on abstract convex spaces. In fact, the study on the KKM theoretic results related certain coercing families on particular types of KKM spaces like H-spaces, L-spaces, FC-spaces, etc. are not necessary, and hence, we concluded that now is the proper time to give up such useless study on L-spaces and their variants FC-spaces.

As a continuation of our [38], the present Part II is a revised and extended version of [34] and shows that some previous or new results generalize or improve corresponding ones in $[1,8,10,16,17]$.

This article is organized as follows:

Section 2 is a routine preliminary on terminology of abstract convex spaces. Here we introduce the KKM maps with respect to a multimap, $\mathfrak{K C}$-maps [resp. $\mathfrak{K O}$-maps], and (partial) KKM spaces. We add a typical general form of the KKM theorem for abstract convex spaces. In Section 3, we begin with a routine diagram showing typical subclasses of abstract convex spaces. Moreover, various subclasses and examples of abstract convex spaces are given for applications of all theorems in this article.

Section 4 is to generalize the coercing families in $[5,8,11,16.17]$. We show that such coercing families can be unified by our condition [I]. In Sections $5-7$, we show that better forms of main theorems in the works on L-spaces can be deduced from a KKM theorem on abstract convex spaces in the sense of [23-27]. Section 5 deals with the KKM type results, and Section 6 with the Fan-Browder type fixed point theorems. Section 7 deals with improvements of minimax inequalities due to the L-space theorists.

Finally, in Section 8, some historical remarks related to L-spaces will be given.

\section{Abstract convex spaces}

Since 2006 we have introduced the concepts of abstract convex spaces, KKM spaces, and partial KKM spaces; see our recent works [27-33] and the references therein. 
Definition 2.1. An abstract convex space $(E, D ; \Gamma)$ consists of a topological space $E$, a nonempty set $D$, and a multimap $\Gamma:\langle D\rangle \multimap E$ with nonempty values $\Gamma_{A}:=\Gamma(A)$ for $A \in\langle D\rangle$, where $\langle D\rangle$ is the set of all nonempty finite subsets of $D$.

For any $D^{\prime} \subset D$, the $\Gamma$-convex hull of $D^{\prime}$ is denoted and defined by

$$
\operatorname{co}_{\Gamma} D^{\prime}:=\bigcup\left\{\Gamma_{A} \mid A \in\left\langle D^{\prime}\right\rangle\right\} \subset E .
$$

A subset $X$ of $E$ is called a $\Gamma$-convex subset of $(E, D ; \Gamma)$ relative to $D^{\prime}$ if, for any $N \in\left\langle D^{\prime}\right\rangle$, we have $\Gamma_{N} \subset X$, that is, $\operatorname{co}_{\Gamma} D^{\prime} \subset X$.

When $D \subset E$, a subset $X$ of $E$ is said to be $\Gamma$-convex if $\operatorname{co}_{\Gamma}(X \cap D) \subset X$; or equivalently, $X$ is $\Gamma$-convex relative to $D^{\prime}:=X \cap D$.

The space is denoted $(E \supset D ; \Gamma)$ when $E \supset D$ and $(E ; \Gamma)$ when $E=D$.

Definition 2.2. Let $(E, D ; \Gamma)$ be an abstract convex space and $Z$ a topological space. For a multimap $F: E \multimap Z$ with nonempty values, if a multimap $G: D \multimap Z$ satisfies

$$
F\left(\Gamma_{A}\right) \subset G(A):=\bigcup_{y \in A} G(y) \quad \text { for all } A \in\langle D\rangle,
$$

then $G$ is called a KKM map with respect to F. A KKM map $G: D \multimap E$ is a KKM map with respect to the identity map $1_{E}$.

A multimap $F: E \multimap Z$ is called a $\mathfrak{K C}$-map [resp. a $\mathfrak{K O}$-map] if, for any closed-valued [resp. open-valued] $K K M$ map $G: D \multimap Z$ with respect to $F$, the family $\{G(y)\}_{y \in D}$ has the finite intersection property. In this case, we denote $F \in \mathfrak{K C}(E, Z)$ [resp. $F \in \mathfrak{K O}(E, Z)$ ].

Some remarks and examples on $\mathfrak{K} \mathfrak{C}$-maps and $\mathfrak{K O}$-maps can be seen in [23, 24]. In this article, we need only the fact that any continuous function $s: E \rightarrow Z$ belongs to $\mathfrak{K C}(E, Z)$.

Definition 2.3. The partial KKM principle for an abstract convex space $(E, D ; \Gamma)$ is the statement $1_{E} \in$ $\mathfrak{K C}(E, E)$; that is, for any closed-valued KKM map $G: D \multimap E$, the family $\{G(y)\}_{y \in D}$ has the finite intersection property. The KKM principle is the statement $1_{E} \in \mathfrak{K C}(E, E) \cap \mathfrak{K D}(E, E)$; that is, the same property also holds for any open-valued KKM map.

An abstract convex space is called a (partial) KKM space if it satisfies the (partial) KKM principle, respectively.

Example 2.1. Consider the following related four conditions for a multimap $G: D \multimap Z$ from a set $D$ into a topological space $Z$ :

(a) $\bigcap_{y \in D} \overline{G(y)} \neq \emptyset$ implies $\bigcap_{y \in D} G(y) \neq \emptyset$.

(b) $\bigcap_{y \in D} \overline{G(y)}=\overline{\bigcap_{y \in D} G(y)}$ ( $G$ is intersectionally closed-valued).

(c) $\bigcap_{y \in D} \overline{G(y)}=\bigcap_{y \in D} G(y)(G$ is transfer closed-valued).

(d) $G$ is closed-valued.

From the definition of $\mathfrak{K C}$-maps, we have a whole intersection property of the Fan type under certain 'coercivity' conditions. The following is given in [28, 30-33]:

Theorem 2.1. Let $(E, D ; \Gamma)$ be an abstract convex space, $Z$ a topological space, $F \in \mathfrak{K C}(E, Z)$, and $G$ : $D \multimap Z$ a multimap such that

(1) $\bar{G}$ is a KKM map w.r.t. F; and

(2) there exists a nonempty compact subset $K$ of $Z$ such that either

(i) $K \supset \bigcap\{\overline{G(y)} \mid y \in M\}$ for some $M \in\langle D\rangle$; or 
(ii) for each $N \in\langle D\rangle$, there exists a $\Gamma$-convex subset $L_{N}$ of $E$ relative to some $D^{\prime} \subset D$ such that $N \subset D^{\prime}, \overline{F\left(L_{N}\right)}$ is compact, and

$$
K \supset \overline{F\left(L_{N}\right)} \cap \bigcap\left\{\overline{G(y)} \mid y \in D^{\prime}\right\}
$$

Then we have

$$
\overline{F(E)} \cap K \cap \bigcap\{\overline{G(y)} \mid y \in D\} \neq \emptyset .
$$

Furthermore,

$(\alpha)$ if $G$ is transfer closed-valued, then $\overline{F(E)} \cap K \cap \bigcap\{G(y) \mid y \in D\} \neq \emptyset$; and

$(\beta)$ if $G$ is intersectionally closed-valued, then $\bigcap\{G(y) \mid y \in D\} \neq \emptyset$.

Remark 2.1. 1. The coercivity (ii) is originated from S. Y. Chang [6] in 1989.

2. Taking $\bar{K}$ instead of $K$, we may assume $K$ is closed and the closure notations in (i) and (ii) can be erased.

3. In [33], we showed that a particular form of Theorem 2.1 for $F=1_{E}$ unifies several important KKM type theorems appeared in history.

4. Many particular forms of Theorem 2.1 have equivalent formulations or lead many KKM theoretic results.

\section{Various subclasses and examples of abstract convex spaces}

The following diagram for triples $(E, D ; \Gamma)$ on abstract convex spaces is well known:

$$
\begin{aligned}
\text { Simplex } & \Longrightarrow \text { Convex subset of a t.v.s. } \Longrightarrow \text { Convex space } \Longrightarrow \text { H-space } \\
& \Longrightarrow \text { G-convex space } \Longrightarrow \phi_{A} \text {-space } \Longrightarrow \text { KKM space } \\
& \Longrightarrow \text { Partial KKM space } \Longrightarrow \text { Abstract convex space. }
\end{aligned}
$$

Example 3.1. We introduce typical subclasses of KKM spaces. Some details can be seen in [25] and the references therein.

(1) Simplex - The 1929 KKM theorem [19] was for the triple $\left(\Delta_{n}, V\right.$; co), where $\Delta_{n}$ is the standard $n$-simplex with vertices $A=\left\{e_{i}\right\}_{i=0}^{n}, V$ the set of vertices and co : $\langle V\rangle \multimap \Delta_{n}$ the convex hull operation.

(2) Convex subsets of a t.v.s. - - The 1961 KKM lemma of Ky Fan (so called the KKMF theorem) [13] was for the triple $(Y \supset X$; co), where $X$ is an arbitrary set in a topological vector space $Y$.

Moreover, every nonempty convex subset $X$ of a topological vector space is a convex space [in the following sense in (3)] with respect to any nonempty subset $D$ of $X$, and the converse is not true.

(3) A convex space $(X, D)=(X, D ; \Gamma)$ is a triple where $X$ is a subset of a vector space, $D \subset X$ such that co $D \subset X$, and each $\Gamma_{A}$ is the convex hull of $A \in\langle D\rangle$ equipped with the Euclidean topology; see [25]. Note that $(X, D)$ can be represented by $(X, D ; \Gamma)$ where $\Gamma:\langle D\rangle \multimap X$ is the convex hull operator. If $X=D$ is convex, then $X=(X, X)$ becomes a convex space in the sense of Lassonde [20].

(4) A triple $(X, D ; \Gamma)$ is called an $H$-space by Park in 1992 if $X$ is a topological space, $D$ a nonempty subset of $X$, and $\Gamma=\left\{\Gamma_{A}\right\}$ a family of contractible (or, more generally, $\omega$-connected) subsets of $X$ indexed by $A \in\langle D\rangle$ such that $\Gamma_{A} \subset \Gamma_{B}$ whenever $A \subset B \in\langle D)$.

If $D=X$, we denote $(X ; \Gamma)$ instead of $(X, X ; \Gamma)$, which is called a $c$-space by Horvath [18] or an H-space by Bardaro and Ceppitelli in 1988.

(5) A generalized convex space or a $G$-convex space $(X, D ; \Gamma)$ is an abstract convex space such that for each $A \in\langle D\rangle$ with the cardinality $|A|=n+1$, there exists a continuous function $\phi_{A}: \Delta_{n} \rightarrow \Gamma(A)$ such that $J \in\langle A\rangle$ implies $\phi_{A}\left(\Delta_{J}\right) \subset \Gamma(J)$, where $\Delta_{J}$ the face of $\Delta_{n}$ corresponding to $J \in\langle A\rangle$; see [21, 25].

When $X=D$, a G-convex space is called an $L$-space; see [4].

(6) A space having a family $\left\{\phi_{A}\right\}_{A \in\langle D\rangle}$ or simply a $\phi_{A^{-}}$space $\left(X, D ;\left\{\phi_{A}\right\}_{A \in\langle D\rangle}\right)$ consists of a topological space $X$, a nonempty set $D$, and a family of continuous functions $\phi_{A}: \Delta_{n} \rightarrow X$ (that is, singular $n$-simplices) for $A \in\langle D\rangle$ with the cardinality $|A|=n+1$. 
Example 3.2. In our previous work [37] and others, we listed known concrete examples of KKM spaces other than the subclasses in the diagram as follows:

(1) Hyperconvex metric spaces of Aronszajn and Panitchpakdi (1956)

(2) Hyperbolic metric spaces - Kirk (1982), Reich and Shafrir (1990)

(3) Topological semilattices - Horvath and Llinares-Ciscar (1996)

(4) E-convex spaces - Youness (1999)

(5) Bayoumi's KKM spaces - Bayoumi (2003)

(6) $\Gamma$-convex spaces - Zafarani (2004)

(7) $\mathbb{R}$-tree - Kirk and Panyanak (2007)

(8) Horvath's convex space - Horvath (2008)

(9) $\mathbb{B}$-spaces - Briec and Horvath (2008)

(10) Connected linearly ordered spaces - Park (2007)

(11) Extended long line $L^{*}$ - Park (2008)

(12) Complete continuous midpoint metric spaces - Horvath (2009)

(13) Metric spaces with global nonpositive curvature (NPC) - Niculescu-Roventa (2009)

(14) R-KKM spaces - Sankar Raj and Somasundaram (2012)

(15) KKM spaces of Chaipunya-Kumam - Chaipunya and Kumam (2015)

(16) Complete finite dimensional Riemannian manifolds - Park (2019)

All theorems in this article can be applied to these spaces.

\section{Generalizations of various coercing families}

In this section, we obtain generalizations of coercing families considered by Ben-El-Mechaiekh et al. [5], Chebbi-Gourdel-Hammami [11], Chebbi [8], Hammami [17], and Gourdel-Hammami [16].

Let us begin with the following particular form of the condition (ii) in Theorem 2.1 with $s G: D \multimap Z$ instead of $G: D \multimap Z$ :

[I] (34]) Let $(E, D ; \Gamma)$ be an abstract convex space, $G: D \multimap E$ a multimap, $Z$ a topological space, and $s: E \rightarrow Z$ a continuous map such that

(C) there exists a nonempty compact subset $K$ of $Z$ such that, for each $N \in\langle D\rangle$, there exists a compact $\Gamma$-convex subset $L_{N}$ of $E$ relative to some $D^{\prime} \subset D$ such that $N \subset D^{\prime}$ and

$$
s\left(L_{N}\right) \cap \bigcap_{y \in D^{\prime}} s G(y) \subset K .
$$

Note that $s \in \mathfrak{K C}(E, Z)$.

Under the situation of [I], we have the following:

Proposition 4.1. If $(E, D ; \Gamma)$ is a (partial) KKM space, then so is the abstract convex space $(Z, D ; s \Gamma)$, where $s \Gamma:\langle D\rangle \multimap Z$.

Proof. Let $G^{\prime}: D \multimap Z$ be a closed-valued KKM map, that is, for any $A \in\langle D\rangle, s \Gamma(A) \subset G^{\prime}(A)$ or $\Gamma(A) \subset\left(s^{-1} G^{\prime}\right)(A)$. Then $s^{-1} G^{\prime}: D \multimap E$ is a closed-valued KKM map on the partial KKM space $(E, D ; \Gamma)$. Hence $\left\{s^{-1} G^{\prime}(a)\right\}_{a \in D}$ has the finite intersection property and so does the family $\left\{G^{\prime}(a)\right\}_{a \in D}$.

Note that the above proof also works for open-valued KKM maps on KKM spaces.

Proposition 4.2. The set $s\left(L_{N}\right)$ is a compact $s \Gamma$-convex subset of $(Z, D ; s \Gamma)$.

Proof. Since $L_{N}$ is compact and $s$ is continuous, $s\left(L_{N}\right)$ is compact. Since $L_{N}$ is $\Gamma$-convex relative to some $D^{\prime} \subset D$ such that $N \subset D^{\prime}$, for any $A \in\left\langle D^{\prime}\right\rangle$, we have $\Gamma(A) \subset L_{N}$ and hence $s \Gamma(N) \subset s\left(L_{N}\right)$. Therefore, $s\left(L_{N}\right)$ is $s \Gamma$-convex relative to $D^{\prime} \subset D$. 
In 2011, Chebbi et al. [11] introduced the notion of coercing family in L-spaces for a given map as follows: [II] ([11]) Let $D$ be an arbitrary set in an L-space $(E, \Gamma), Z$ a topological space, and $s: E \rightarrow Z$ a continuous map. A family $\left\{\left(C_{a}, K\right)\right\}_{a \in E}$ is said to be L-coercing for a map $F: D \multimap Z$ with respect to $s$ if

(i) $K$ is a compact subset of $Z$;

(ii) for each $N \in\langle D\rangle$, there exists a compact L-convex set $L_{N}$ in $E$ containing $N$ such that

$$
x \in L_{N} \Rightarrow C_{x} \cap D \subset L_{N} \cap D
$$

(iii) $\left\{x \in E \mid s(x) \in \bigcap_{y \in C_{x} \cap D} F(y)\right\} \subset s^{-1}(K)$.

Here $C_{x}$ is an L-compact subset of $E$ for each $x \in D$. Note that $(E, D ; \Gamma)$ is a G-convex space, contrary to the intention of the so-called L-space theorists.

Proposition 4.3. $[\mathrm{II}] \Longrightarrow[\mathrm{I}]$.

Proof. Under the situation of [II], note that $(E \supset D ; \Gamma)$ is a G-convex space and hence a (partial) KKM space. Let $G:=s^{-1} F: D \multimap E$ and, for any $N \in\langle D\rangle$, we have a compact $\Gamma$-convex subset $L_{N}$ of $E$ containing $N$. Choose an $x \in L_{N}$ and let $D^{\prime}:=\left(C_{x} \cap D\right) \cup N \subset L_{N} \cap D$ by (ii) (and Remark 1 in [7]). Then $L_{N}$ is $\Gamma$-convex relative to $D^{\prime} \subset D$ containing $N$. Moreover, by (iii),

$$
x \in \bigcap_{y \in D^{\prime}} G(y) \subset \bigcap_{y \in C_{x} \cap D} s^{-1} F(y) \subset s^{-1}(K) .
$$

Hence

$$
s(x) \in s\left(L_{N}\right) \cap \bigcap_{y \in D^{\prime}} s G(y) \subset K .
$$

Therefore [I] holds.

Motivated by [5], we define the following:

[III] Let $(E, D ; \Gamma)$ be an abstract convex space and $Z$ a topological space. We say that a map $F: D \multimap Z$ has a coercing family $\left\{\left(D_{i}, K_{i}\right)\right\}_{i \in I}$ if and only if

(1) for each $i \in I, K_{i}$ is a compact subset of $Z$ and $D_{i} \subset D$ such that, for each $N \in\langle D\rangle$, there exist a compact subset $L_{N}^{i}$ of $E$ that is $\Gamma$-convex relative to $D_{i} \cup N$;

(2) for each $i \in I$, there exists $k \in I$ with $\bigcap_{y \in D_{k}} F(y) \subset K_{i}$.

Proposition 4.4. $[\mathrm{III}] \Longrightarrow[\mathrm{I}]$.

Proof. Let $G: D \multimap E$ and $s: E \rightarrow Z$ be given in [I] and let $F=s G: D \multimap Z$. Choose any $i \in I$ by [III](1), we have $K_{i}$ and $D_{i}$ such that, for each $N \in\langle D\rangle$, there exists a compact $\Gamma$-convex subset $L_{N}:=L_{N}^{i}$ of $E$ relative to $D^{\prime}=D_{i} \cup N$. By [III](2), we have a $k \in I$ such that

$$
\bigcap_{y \in D_{k}} F(y)=\bigcap_{y \in D_{k}} s G(y) \subset K_{i} .
$$

Since $i$ was arbitrary, we may assume $k=i$ and $K=K_{i}$. Moreover, since $D^{\prime}=D_{k} \cup N$, we have

$$
\bigcap_{y \in D^{\prime}} s G(y) \subset K \Longrightarrow s\left(L_{N}\right) \cap \bigcap_{y \in D^{\prime}} s G(y) \subset K .
$$

Hence the coercivity condition [I] holds. 
The definition [III] improves the following coercivity in the sense of Ben-El-Mechaiekh, Chebbi, and Florenzano [[5], Definition 2.1]:

[IV] ([5]) Consider a subset $X$ of a Hausdorff topological vector space $E$ and a topological space $Z$. A family $\left\{\left(D_{i}, K_{i}\right)\right\}_{i \in I}$ of pairs of sets is said to be coercing for a map $F: X \multimap Z$ if and only if:

(i) for each $i \in I, D_{i}$ is contained in a compact convex subset of $X$, and $K_{i}$ is a compact subset of $Z$;

(ii) for each $i, j \in I$, there exists $k \in I$ such that $D_{i} \cup D_{j} \subset D_{k}$;

(iii) for each $i \in I$, there exists $k \in I$ with $\bigcap_{x \in D_{k}} F(x) \subset K_{i}$.

If $I$ is a singleton, the family is called a single coercing family. Note that $(E, X ; c o)$ is a G-convex space and that (ii) will be shown redundant.

Remark 4.1. In [5], it is noted that the condition (iii) holds if and only if the 'dual' map $\Phi: Z \multimap X$ of $F$, defined by $\Phi(z)=X \backslash F^{-}(z), z \in Z$ verifies

(iii) $^{\prime} \forall i \in I, \exists k \in I, \forall z \in Z \backslash K_{i}, \quad \Phi(z) \cap C_{k} \neq \emptyset$.

In [5], there are given several deep examples of condition (iii)' related to an exceptional family, an escaping sequence, an attracting trajectory, and others.

Remark 4.2. In [11], it is shown that L-coercing families in [II] contain coercing families in the sense of [IV].

Here we show that [IV] is a particular case of the coercivity [III] for abstract convex spaces:

Proposition 4.5. [IV] $\Longrightarrow$ [III].

Proof. Since each $D_{i}$ is contained in a compact convex subset of $X \subset E$ by [IV](i) and $E$ is a Hausdorff topological vector space, for each $N \in\langle X\rangle$, there exists a compact convex subset $L_{N}^{i}$ of $E$ containing $D_{i} \cup N$; see Lassonde [20]. Therefore, Condition [III](1) holds. Since [IV](iii) is the same to [III](2), all requirements of [III] are satisfied.

Note that Condition [IV](ii) is redundant for [III]. Note that all of [I]-[IV] are examples of the coercivity (ii) in Theorem 2.1.

From now on we add up new things not treated in [34].

In 2006, Chebbi [8] introduced the following generalized form of [IV] where $\mathrm{H}$-spaces are $c$-spaces due to Horvath [18]:

[V] ([8]) Let $(X, \Gamma)$ be an H-space and $Y$ a topological space. A family $\left\{\left(C_{i}, K_{i}\right)\right\}_{i \in I}$ is said to be H-coercing for a correspondence $F: X \rightarrow Y$ if and only if:

(i) For each $i \in I, C_{i}$ is an H-compact subset of $X$ and $K_{i}$ is a compact subset of $Y$;

(ii) For each $i, j \in I$, there exists $k \in I$ such that $C_{i} \cup C_{j} \subseteq C_{k}$;

(iii) For each $i \in I$, there exists $k \in I$ such that:

$$
\bigcap_{x \in C_{k}} F(x) \subseteq K_{i}
$$

Proposition 4.6. $[\mathrm{I}] \Longleftarrow[\mathrm{III}] \Longleftarrow[\mathrm{V}] \Longleftarrow[\mathrm{IV}]$.

The following was adopted by Hammami [17] in 2007 and Gourdel-Hammami [16] in 2007, and originated from Chebbi-Gourdel-Hammami [11] in 2011:

[VI] ([17]) Let $Z$ be an arbitrary set of an L-space $(X, \Gamma), Y$ a topological space, and $s: X \rightarrow Y$ a continuous map. A family $\left\{\left(C_{a}, K\right)\right\}_{a \in X}$ is said to be L-coercing for a correspondence $F: Z \multimap Y$ with respect to $s$ if and only if: 
(i) $K$ is a quasi-compact subset of $Y$,

(ii) for each $A \in\langle Z\rangle$, there exists a quasi-compact L-convex set $D^{A}$ in $X$ containing $A$ such that:

$$
x \in D^{A} \Longrightarrow C_{x} \cap Z \subset D^{A} \cap Z,
$$

(iii) $\left\{y \in Y \mid y \in \bigcup_{z \in s^{-1}(y)} \bigcap_{x \in C_{z} \cap Z} F(x)\right\} \subset K$.

Note that $(X, Z ; \Gamma)$ is a $\mathrm{G}$-convex space.

Hammami [17] added: For more explanation of the L-coercivity and to see that this coercivity can't be compared to the coercivity in the sense of Ben-El-Mechaiekh, Chebbi and Florenzano in [5], see [11].

However, we note the following:

Proposition 4.7. [I] $\Longleftarrow[\mathrm{II}] \Longleftrightarrow[\mathrm{VI}]$.

Therefore all coercing families due to the L-space theorists are consequences of Theorem 2.1(ii). Moreover, Conditions [II], [IV], [V], and [VI] are stated for G-convex spaces, and hence their inventors —- ChebbiGourdel-Hammami [11], Ben-El-Mechaiekh - Chebbi-Florenzano [5], Chebbi [8], Hammami [17], and GourdelHammami [16] are all ignorant of G-convex spaces. This is a proper evidence that they did not seriously read any works on G-convex spaces.

\section{Generalization of the KKM principle}

In this section, we deduce generalized better forms of KKM type theorems on the L-spaces in [1, 5, 17, 8, [10, 11, 16, 17] from our KKM Theorem 2.1 on abstract convex spaces.

In fact, the following includes a large number of results on L-spaces:

Theorem 5.1. ([34]) Let $(E, D ; \Gamma)$ be an abstract convex space, $Z$ an arbitrary topological space and $G$ : $D \multimap Z$ a closed-valued multimap. Suppose that there exists a continuous map $s: E \rightarrow Z$ such that:

(1) the multimap $R: D \multimap E$ defined by $R(y):=s^{-1}(G(y))$ is $K K M$;

(2) the coercivity condition $[\mathrm{I}]$ holds for $R$ instead of $G$.

Then we have $K \cap \bigcap_{y \in D} G(y) \neq \emptyset$.

Proof. We apply Theorem 2.1 with $F=s$.

(1) Since $s^{-1} G$ is a closed-valued KKM map, Condition (1) implies $\Gamma_{A} \subset R(A)=s^{-1} G(A)$ and $s \Gamma_{A} \subset$ $s R(A)=G(A)$ for all $A \in\langle D\rangle$. Therefore $\bar{G}$ is a KKM map w.r.t. $s$.

(2) Condition (2) implies (ii) in Theorem 2.1 with $F=s$ and $G=s R$. Therefore, by the case (ii) of Theorem 2.1, we have

$$
s(E) \cap K \cap \bigcap_{y \in D} s R(y) \neq \emptyset .
$$

This implies the conclusion.

The main theorem of [[11], Theorem 1] and others are the particular case of Theorem 5.1 under the assumption of [II] as follows:

Corollary 5.1. ([11, 16, 17]) Let $D$ be an arbitrary set in the $L$-space $(E ; \Gamma), Z$ an arbitrary topological space and $F: D \multimap Z$ a map with quasi-compactly closed values. Suppose that there exists a continuous function $s: E \rightarrow Z$ such that:

(1) the map $R: D \multimap E$ defined by $R(y)=s^{-1}(F(y))$ is $K K M$;

(2) there exists an L-coercing family for $F$ with respect to $s$ as in [II]. Then $K \cap \bigcap_{x \in D} F(x) \neq \emptyset$.

The main theorem [[5], Theorem 3.1.] of [5] is the particular case $s=1_{Y}$ of Theorem 5.1 under the assumption of [IV] as follows: 
Corollary 5.2. ([5]) Let $E$ be a Hausdorff topological vector space, $Y$ a convex subset of $E, X$ a non-empty subset of $Y$, and $F: X \multimap Y$ a KKM map with compactly closed (in $Y$ ) values. If $F$ admits a coercing family in the sense of [IV], then $\bigcap_{x \in X} F(x) \neq \emptyset$.

Remark 5.1. From the above corollaries, we notice the following:

1. The quasi-compactly closed sets are compactly closed sets in modern usage and can be replaced by mere closed sets by adopting compactly generated extension (like $k$-spaces) of the original topology.

2. Our proofs are based on Theorem 2.1 and different from that of [5, 7].

3. In view of [III], condition (ii) in [IV] of a coercing family is redundant.

4. The existence of a coercing family [IV] is simply equivalent to that of a single coercing family.

5. In Corollaries 5.1 and 5.2, F can be transfer closed-valued or intersectionally closed-valued.

6. As was noted in [5], if the coercing family is single, then Corollary 5.2 reduces to the $1984 \mathrm{KKM}$ theorem of Fan [[15], Theorem 4] which in turn generalizes the KKMF principle.

We add up some more consequences of Theorem 5.1

As in [17, for any correspondence $F: X \multimap Y$, let $F^{*}: Y \multimap X$ be the 'dual' correspondence of $F$ defined, for all $y \in Y$, by $F^{*}(y)=X \backslash F^{-1}(y)$, where $F^{-1}(y)=\{x \in X \mid y \in F(x)\}$.

The following consequence [[16], Theorem 1.2] of Corollary 5.1 was used in order to generalize Fan's minimax inequality:

Corollary 5.3. ([16]) Let $(X ; \Gamma)$ be an L-space, $Y$ an arbitrary topological space and $F, G: X \multimap Y$ be two correspondences satisfying:

(a) for every $x \in X, F(x)$ is strongly compactly closed,

(b) for every $x \in X, G(x) \subset F(x)$,

(c) there exists a continuous function $s: X \rightarrow Y$ such that:

1. for every $x \in X, s(x) \in G(x)$,

2. for every $x \in X, S^{*}(x)$ where $S$ is defined by $S(x)=s^{-1}(G(x))$ is L-convex,

3. there exists an L-coercing family $\left\{\left(C_{x}, K\right)\right\}_{x \in X}$ for $F$ with respect to $s$.

Then $\bigcap_{x \in Z} F(x) \neq \emptyset$.

Proof. By replacing the topology of $X$ by the strongly compactly generated one, $F$ has simply closed values. In order to apply Corollary 5.1, it suffices to show that the correspondence $R: X \multimap X$ defined by $R(x)=$ $s^{-1}(F(x))$ is KKM. Let $A \in\langle X\rangle$ and $z \in \Gamma(A)$, then by (c.1), $s(z) \in G(\Gamma(A))$. One can check that Condition (c.2) can be equivalently rewritten as $S(\Gamma(A)) \subset S(A)$. Moreover, by (c.1), for all $B \subset X, B \subset S(B)$, in particular $\Gamma(A) \subset S(\Gamma(A))$. Hence we obtain $\Gamma(A) \subset S(A)$. By construction, $S \subset R$, which implies that $R$ is KKM.

The following main result of Chebbi [[8], Theorem 3.1] is the particular case of Corollary 5.3 since H-spaces are L-spaces:

Corollary 5.4. ([8]) Let $(X, \Gamma)$ be an $H$-space, $Y$ any topological space and $F: X \rightarrow Y$ a correspondence such that:

(1) For every $x \in X, F(x)$ is compactly closed in $X$.

(2) For some continuous map $s: X \rightarrow Y$ the correspondence $G: X \rightarrow X$ given by $G(x)=s^{-1}(F(x))$ is $H-K K M$.

(3) There exists an H-coercing family $\left\{\left(C_{i}, K_{i}\right)\right\}_{i \in I}$ for $F$.

Then $\bigcap_{x \in X} F(x) \neq \emptyset$.

Proof. By switching the topology of $X$ to the compactly generating one (as in $k$-spaces), all $F(x)$ becomes simply closed in (1). Since H-spaces are KKM spaces, the closed-valued KKM map $G$ in (2) has the finite intersection property for its values. The condition (3) is an abstract form of [IV] in Section 3. Since [IV] implies [I], The conclusion follows from Theorem 5.1. 
The following [[8], Theorem 3.2] is a simple consequences of the preceding one:

Corollary 5.5. ([8]) Let $(X, \Gamma)$ be an H-space and $F, G: X \rightarrow X$ two correspondences such that:

(a) For every $x \in X, G(x)$ is compactly closed and $F(x) \subset G(x)$.

(b) For every $x \in X, x \in F(x)$.

(c) For every $x \in X, F^{*}(x)$ is H-convex .

(d) There exists an H-coercing family $\left\{\left(C_{i}, K_{i}\right)\right\}_{i \in I}$ for $F$.

Then $\bigcap_{x \in X} G(x) \neq \emptyset$.

In 8 ] this was used to prove results on minimax inequalities for Riesz spaces. Our original G-convex space paper [38] was the origin of L-spaces, and is quoted in [8] for more details about generalized convexity.

The following extends Theorem 5.1 for the case $E \supset D$ and the main result of Hammami [[17], Theorem 1.1] in 2007:

Theorem 5.2. Let $(E \supset D ; \Gamma)$ be an abstract convex space, $Y$ an arbitrary topological space and and $F, G: D \multimap Y$ two correspondences such that:

(a) for every $x \in D, F(x)$ is closed,

(b) for every $x \in D, G(x) \subset F(x)$,

(c) there is a continuous function $s: E \rightarrow Y$ satisfying:

1. the correspondence $R: D \multimap E$ defined by $R(x)=s^{-1}(F(x))$ is $K K M$,

2. the coercivity condition [I] holds for $s^{-1} G$.

3. for each compact $\Gamma$-convex set $C$ in $E$ :

$$
\bigcap_{x \in C \cap D} G(x) \cap s(C) \neq \emptyset \Longleftrightarrow \bigcap_{x \in C \cap D} F(x) \cap s(C) \neq \emptyset
$$

Then we have $K \cap \bigcap_{x \in D} F(x) \neq \emptyset$.

Note that Theorem 5.2 for $F=G$ reduces to Theorem 5.1 for the case $E \supset D$ and that $s: E \rightarrow Y$ belongs to $\mathfrak{K C}(E, Y)$

Proof. Since each $F(x)$ is closed, it suffices to show that for each finite subset $N$ of $D, \bigcap_{x \in N} F(x) \cap K \neq \emptyset$.

Let $N \in\langle D\rangle$. Since (c.2) implies condition $[\mathrm{I}](\mathrm{C})$ for $s^{-1} G$, there exists a compact $\Gamma$-convex subset $L_{N}$ of $E$ relative to some $D^{\prime} \subset D$ such that $N \subset D^{\prime}$ and

$$
s\left(L_{N}\right) \cap \bigcap_{y \in D^{\prime}} G(y) \subset K .
$$

So, we may assume $D^{\prime}=L_{N} \cap D$.

Consider now the correspondence $R^{N}: D^{\prime} \multimap L_{N}$ defined by $R^{N}(x)=s^{-1} F(x) \cap L_{N}$ for $x \in D^{\prime}$. By Hypothesis (c.1) and the $\Gamma$-convexity of $L_{N}$, it is immediate that the correspondence $R^{N}$ is a KKM map. Next, by the continuity of $s, F(x) \cap s\left(L_{N}\right)$ is closed in $s\left(L_{N}\right)$, and then $R^{N}(x)=s_{0}^{-1}\left(F(x) \cap s\left(L_{N}\right)\right)$, where $s_{0}$ is the restriction of $s$ to $L_{N}$, is closed in $L_{N}$ and consequently $R^{N}(x)$ is compact. Since $\left(L_{N}, D^{\prime} ;\left.\Gamma\right|_{D^{\prime}}\right)$ is also an abstract convex space and $\left.s\right|_{L_{N}} \in \mathfrak{K C}\left(L_{N}, Y\right)$, we deduce by Theorem 2.1(i) that $\bigcap_{x \in D^{\prime}} R^{N}(x) \neq \emptyset$. Since $s\left(R^{N}(x)\right) \subset F(x) \cap s\left(L_{N}\right)$ for all $x \in D^{\prime}$, we have $\bigcap_{x \in D^{\prime}} F(x) \cap s\left(L_{N}\right) \neq \emptyset$. Then by (c.3) with $C=L_{N}, \bigcap_{x \in D^{\prime}} G(x) \cap s\left(L_{N}\right) \neq \emptyset$. To finish the proof, we will show that

$$
\bigcap_{x \in D^{\prime}} G(x) \cap s\left(L_{N}\right) \subset \bigcap_{x \in N} F(x) \cap K
$$

with $D^{\prime}=L_{N} \cap D$. Indeed, it is clear by (b) that $\bigcap_{x \in L_{N} \cap D} G(x) \cap s\left(L_{N}\right) \subset \bigcap_{x \in N} F(x)$, and by the conclusion of [I](C) for $s^{-1} G$ we have $\bigcap_{x \in L_{N} \cap D} G(x) \cap s\left(L_{N}\right) \subset K$. Hence, the theorem is proved. 
The main result of Hammami [[17], Theorem 1.1] in 2007 is the following consequence of Theorem 5.2

Corollary 5.6. ([17]) Let $Z$ be an arbitrary set in the L-space $(X ; \Gamma), Y$ an arbitrary topological space and $F, G: Z \multimap Y$ two correspondences such that:

(a) for every $x \in Z, F(x)$ is strongly compactly closed,

(b) for every $x \in Z, G(x) \subset F(x)$,

(c) there is a continuous function $s: X \rightarrow Y$ satisfying:

1. the correspondence $R: Z \multimap X$ defined by $R(x)=s^{-1}(F(x))$ is $L-K K M$,

2. there exists an L-coercing family $\left\{\left(C_{a}, K\right)\right\}_{a \in X}$ for $G$ with respect to $s$,

3. for each quasi-compact $L$-convex set $C$ in $X$ :

$$
\bigcap_{x \in C \cap Z} G(x) \cap s(C) \neq \emptyset \Longleftrightarrow \bigcap_{x \in C \cap Z} F(x) \cap s(C) \neq \emptyset
$$

Then $\bigcap_{x \in Z} F(x) \neq \emptyset$; more precisely $K \cap \bigcap_{x \in Z} F(x) \neq \emptyset$.

Proof. Note that $(X \supset Z ; \Gamma)$ is a G-convex space and hence an abstract convex space, and $F$ is closed-valued by switching the topology of $X$ to the strongly compactly generated topology (as for $k$-spaces). Moreover, $R=s^{-1} F$ is KKM and Condition (c.2) implies the coercivity condition [I] for $R=s^{-1} G$ by Proposition 4.3 Then by Theorem 5.2, we have the conclusion.

Comments on Corollary 5.6 .

(1) Corollary 5.6 is extremely artificial and its usefulness is doubtful because of the following observations.

(2) Note that $(X \supset Z ; \Gamma)$ is an abstract convex space. More precisely it is a G-convex space, contrary to the routine claim of the L-space theorists. Traditionally L-spaces are pairs not triples; see our [38].

(3) Switching the topology of $Y$ to compactly generated one (like $k$-spaces), each $T(x)$ can be simply closed.

(4) Existence of L-coercing family is the same to [II] which implies the more reasonable ordinary coercivity [I] in Section 4. In fact, Condition (ii) of Theorem 2.1 is the origin of [I] and has scores of applications in the KKM theory.

(5) The author gives no example of his own L-coercing family.

(6) The author should give an example satisfying (c.3) and other requirements.

The following is rather artificial KKMF theorem:

Corollary 5.7. ([11]) Let $(X, \Gamma)$ be an L-space, $Z$ a nonempty subset of $X$ and $F: Z \multimap X$ an $L$-KKM correspondence with strongly compactly closed values. Suppose that for some $z \in Z$, the correspondence $F(z)$ is quasi-compact, then $\bigcap_{x \in Z} F(x) \neq \emptyset$.

Note that $(X \supset Z ; \Gamma)$ is a G-convex space.

In 2018, Altwaijry-Ounaies-Chebbi [1] claimed that the KKMF principle can be extended to L-spaces as follows:

Corollary 5.8. ([1]) Let $(E, \Gamma)$ be an L-space, $X$ a nonempty subset of $E$ and $F: X \rightarrow E$ a set-valued map satisfying:

(1) For all $x \in X, F(x)$ is a closed subset of $E$.

(2) For all $A \in\langle E\rangle, \Gamma(A) \subset \bigcup_{x \in A} F(x)$.

(3) For some $z \in X, F(z)$ is compact.

Then $\bigcap_{x \in X} F(x) \neq \emptyset$.

Proof. This is a simple consequence of Theorem 2.1(i). Moreover, this is one of the simplest cases of Corollary 5.6 when

$$
\begin{gathered}
(X, Z, \Gamma)=(E, X ; \Gamma), R=F=G, Y=E, s=1_{E}, I=X, \\
C_{a}=\{a\} \forall a \in I=X, \text { and } K=F(z) \text { in }(3) .
\end{gathered}
$$

Then the conclusion follows from Corollary 5.6 
Remark 5.2. 1. The authors of [1] gave a proof using the KKM theorem and stated that this generalizes several well-known previous theorems. However, this is already known and they did not mention any of plenty of proper generalizations of this theorem.

2. Note that Theorems 5.1 and 5.2 can be applied to all subclasses of abstract convex spaces in Section 4. Recall that, for the last two decades, the L-space theorists have insisted that their L-spaces $(X, \Gamma)$ generalize our generalized (G-convex) spaces $(E, D ; \Gamma)$; and could not recognize the existence of various types of extensions of their obsolete L-spaces such as G-convex spaces, $\phi_{A^{-}}$-spaces, KKM spaces, partial KKM spaces, and abstract convex spaces; see [38], where we declared the fall of L-spaces.

\section{Generalizations of the Fan-Browder fixed point theorems}

In [34, we deduced the better form of the Fan-Browder fixed point theorems in [5] and [11] from the KKM theorem 2.1 on abstract convex spaces. In this section, we add up more better forms of the theorems.

Theorem 6.1. ([34]) $(E, D ; \Gamma)$ be a partial $K K M$ space, and $S: E \multimap D, T: E \multimap E$ maps satisfying

(1) $S^{-}(y)$ is open for each $y \in D$;

(2) $T(x) \supset \operatorname{co}_{\Gamma} S(x)$ for each $x \in E$.

Suppose that there exists a nonempty compact subset $K$ of $E$ satisfying

(3) for each $N \in\langle D\rangle$, there exist $D^{\prime} \subset D$ containing $N$ and a compact $\Gamma$-convex subset $L_{N}$ of $E$ relative to $D^{\prime}$ such that

$$
L_{N} \cap \bigcap\left\{E \backslash S^{-}(y) \mid y \in D^{\prime}\right\} \subset K .
$$

Then either (a) $S$ has a maximal element $x_{0} \in K$, that is, $S\left(x_{0}\right)=\emptyset$; or (b) $T$ has a fixed point $x_{1} \in E$, that is, $x_{1} \in T\left(x_{1}\right)$.

Proof. Suppose $T$ has no fixed point. Define a map $G: D \multimap E$ by

$$
G(y):=E \backslash S^{-}(y)=\{x \in E \mid y \notin S(x)\}, \quad y \in D .
$$

Then $G$ is closed-valued. Moreover, $G$ is a KKM map.

In fact, suppose on the contrary that there exists an $N \in\langle D\rangle$ such that $\Gamma_{N} \not \subset G(N)$; that is, there exists an $x \in \Gamma_{N}$ such that $x \notin G(y)$ for all $y \in N$. In other words, $N \in\left\langle D \backslash G^{-}(x)\right\rangle$ and

$$
y \notin G^{-}(x) \Leftrightarrow x \notin G(y)=E \backslash S^{-}(y) \Leftrightarrow x \in S^{-}(y) \Leftrightarrow y \in S(x)
$$

for all $y \in N$. Hence $N \subset S(x)$ and, by (2), we have $x \in \Gamma_{N} \subset T(x)$. This is a contradiction.

Note that (3) implies condition (ii) of Theorem 2.1 with $E=D$ and $s=1_{E} \in \mathfrak{K C}(E, D, E)$ since $(E, D ; \Gamma)$ is a partial KKM space. Therefore, by Theorem 2.1, we have $K \cap \bigcap_{y \in D} G(y) \neq \emptyset$.

Then we have an $x_{0} \in K$ and $x_{0} \in G(y)=E \backslash S^{-}(y)$ or $y \notin S\left(x_{0}\right)$ for all $y \in D$. Hence $S$ has a maximal element $x_{0} \in K$.

From Theorem 6.1] we obtain a fixed point result [[5], Theorem 3.2] as a Corollary:

Corollary 6.1. ([5]) Let $X$ be a non-empty convex subset of a Hausdorff topological vector space and let $\Phi: X \multimap X$ be a map with open fibers (in $X$ ) and non-empty values. If $\Phi$ admits a single coercing family in the sense of $[\mathrm{IV}]$ satisfying (iii)', then the map $\operatorname{co}(\Phi)$ has a fixed point.

Proof. We will use Theorem 6.1 with $E=D=X, \Gamma=\operatorname{co}, \mathrm{S}=\Phi, \mathrm{T}=\operatorname{co}(\Phi)$. Since $\Phi$ has non-empty values, it does not have a maximal element. Now it suffices to show that (iii)' implies condition (3) of Theorem 6.1.

Suppose $K$ is a compact subset of $X$ and $C$ is contained in a compact convex subset $L$ of $X$. Let $N \in\langle X\rangle$. Since $X$ is a convex subset of a Hausdorff topological vector space, there exists a compact convex subset $L_{N}$ of $X$ containing $D^{\prime}:=L \cup N$. Note that $\Phi(x) \cap D^{\prime} \supset \Phi(x) \cap C \neq \emptyset$ for all $x \in X \backslash K$ by (iii)', that is,

$$
x \in X \backslash K \Rightarrow \Phi(x) \cap D^{\prime} \neq \emptyset \Rightarrow \exists y \in \Phi(x) \cap D^{\prime} \Rightarrow x \in \Phi^{-}(y), \exists y \in D^{\prime} .
$$


Now we have

$$
x \in L_{N} \cap \bigcap_{y \in D^{\prime}}\left(X \backslash \Phi^{-}(y)\right) \Rightarrow x \in X \backslash \Phi^{-}(y), \forall y \in D^{\prime} \Rightarrow x \notin \Phi^{-}(y), \forall y \in D^{\prime} .
$$

Therefore, $x \notin X \backslash K$ and hence $x \in K$. So condition (3) of Theorem 6.1 holds.

The following equivalent variant of Theorem 6.1 is given as [[34], Theorem 5.3]:

Theorem 6.2. ([34]) Let $(E, D ; \Gamma)$ be a partial KKM space, $Z$ an arbitrary topological space and $G: D \multimap Z$, $H: E \multimap Z$ multimaps. Suppose that there exists a continuous map $s: E \rightarrow Z$ such that:

(1) $G(y)$ is open for each $y \in D$;

(2) $s^{-1} H(x) \supset \operatorname{co}_{\Gamma} G^{-} s(x)$ for each $x \in E$.

Suppose that there exists a nonempty compact subset $K$ of $E$ satisfying

(3) for each $N \in\langle D\rangle$, there exist $D^{\prime} \subset D$ containing $N$ and a compact $\Gamma$-convex subset $L_{N}$ of $E$ relative to $D^{\prime}$ such that

$$
s\left(L_{N}\right) \cap \bigcap\left\{Z \backslash s^{-1} G(y) \mid y \in D^{\prime}\right\} \subset K .
$$

Then either (a) $G^{-} s$ has a maximal element $x_{0} \in E$, that is, $G^{-} s\left(x_{0}\right)=\emptyset$; or (b) $s^{-1} H$ has a fixed point $x_{1} \in E$, that is, $s\left(x_{1}\right) \in H\left(x_{1}\right)$.

Proof. In view of Propositions 4.1 and $4.2,(Z, D ; s \Gamma)$ is a partial KKM space and $s\left(L_{N}\right)$ is a compact $s \Gamma$-convex subset relative to $D^{\prime}$. We apply Theorem 6.1 replacing $(E, D ; \Gamma)$ by $(Z, D ; s \Gamma), S:=G^{-} s$ and $T:=s^{-1} H$. Then

(1) $S^{-}=s^{-1} G$ is open-valued since so is $G$ and $s$ is continuous.

(2) $T(x)=s^{-1} H(x) \supset \mathrm{co}_{\Gamma} \mathrm{G}^{-} \mathrm{s}(\mathrm{x})=\mathrm{co}_{\Gamma} \mathrm{S}(\mathrm{x})$ for each $x \in E$.

(3) Condition (3) of Theorem 6.1 with $S^{-}=s^{-1} G$ holds.

Therefore, by Theorem6.1, the conclusion follows.

Remark 6.1. Note that we deduced Theorem 6.2. from Theorem 6.1. Conversely, Theorem 6.2 for $E=Z$ and $s=1_{E}$ reduces to Theorem 6.1.

As a corollary of Theorem 6.2, we obtain [[11], Theorem 2].

Corollary 6.2. ([11]) Let $(X ; \Gamma)$ be an L-space, $Z$ an arbitrary topological space, $s: X \rightarrow Z$ a continuous map and $S: X \multimap Z$ a multimap such that:

(i) for each $x \in X, S(x)$ is quasi-compactly open in $Z$;

(ii) for each $z \in Z, S^{-1}(z)$ is nonempty and L-convex;

(iii) there exists an L-coercing family $\left\{\left(C_{x}, K\right)\right\}_{x \in X}$ for the map $Q(x)=Z \backslash S(x)$ with respect to s.

Then there exists $x_{0} \in X$ such that $s\left(x_{0}\right) \in S\left(x_{0}\right)$. In particular, if $s$ is the identity map, then $S$ has a fixed point.

Proof. Put $E=D=X$ and $G=H=S$ in Theorem 6.2

As was noted in [5], Theorems in Sections 5 and 6 can be used to extend existing results on various equilibrium problems, solvability of complementarity problems, existence of zero on non-compact domains, and existence of equilibria for qualitative games and abstract economies.

We have another variant of Corollary 6.2 given by Chebbi [[8], Proposition 4.1] as follows:

Corollary 6.3. ([8]) Let $(X, \Gamma)$ be an $H$-space, $Y$ a topological space and $S: X \rightarrow Y$ a correspondence such that:

(i) For each $x \in X, S(x)$ is compactly open in $Y$.

(ii) For each $y \in X, S^{-1}(y)$ is nonempty and H-convex. 
(iii) There exists an H-coercing family $\left\{\left(C_{i}, K_{i}\right)\right\}_{i \in I}$ for the correspondence $F: X \rightarrow Y$ defined by $F(x)=Y \backslash S(x), \forall x \in X$.

Then, for each continuous function $s: X \rightarrow Y$, there exists an $x_{0} \in X$ such that $s\left(x_{0}\right) \in S\left(x_{0}\right)$. In particular, $S$ has a fixed point.

The last conclusion should be "In particular, if $X=Y$ and $s=1_{X}$, then $S$ has a fixed point."

Corollary 6.3 was also given as Chebbi [[10], Proposition 3.1] in 2015. The following result of Chebbi [[10], Proposition 3.2] is a geometrical formulation of Corollary 6.3.

Corollary 6.4. ([10]) Let $(X, \Gamma)$ be an $H$-space, $Y$ a topological space, $Z$ an arbitrary set and $s: X \rightarrow Y$ a continuous function. Let $A \subset Z$ and $g: X \times Y \rightarrow Z$ be a function such that:

(a) For every $x \in X$, the set $\{y \in Y \mid g(x, y) \in A\}$ is compactly open.

(b) For every $y \in Y$, the set $\{x \in X \mid g(x, y) \in A\}$ is nonempty and H-convex.

(c) There exists a family $\left\{\left(C_{i}, K_{i}\right)\right\}_{i \in I}$ satisfying conditions (i) and (ii) of [V] and the following one: For all $i \in I$, there exist $k \in I$ such that $\left\{y \in Y \mid g(x, y) \notin A \forall x \in C_{k}\right\} \subset K_{i}$.

Then $g$ satisfies at least one of the following properties:

(1) There exists $\hat{y} \in Y$ such that $g(x, \hat{y}) \notin A$ for all $x \in X$.

(2) There is $\hat{x} \in X$ such that $g(\hat{x}, s(\hat{x})) \in A$.

As in the case of classical convexity, we can deduce from Corollary 5.8 the following Fan-Browder type fixed point result [[1], Proposition 3.2]:

Corollary 6.5. ([1]) Let $X$ be a nonempty compact L-convex subset of an L-space $(E, \Gamma), F, G: X \multimap X$ two set-valued maps satisfying the following:

(i) For each $x \in X, F(x) \neq \emptyset$ and $F(x) \subset G(x)$.

(ii) For each $x \in X, G(x)$ is L-convex.

(iii) For each $y \in X, F^{-1}(y)$ is open in $X$.

Then $G$ admits a fixed point, that is a point $\hat{x} \in X$ such that $\hat{x} \in G(\hat{x})$.

The authors of [1] gave a proof using their KKM theorem and stated that this generalizes several wellknown previous theorems. However, they did not mention any of so many proper generalizations of this theorem.

Then the authors continue: "The concept of KF-majorization due to Borglin and Keiding is then easily extended to L-spaces and a result on the existence of a maximal element for such correspondence is deduced. As an application, we prove an equilibrium existence result for qualitative games defined in an L-space and an equilibrium result for an abstract economy."

Note that such arguments were already given by many authors for much more general convexity structure than L-structures.

\section{On general minimax inequalities and applications}

It is well-known that any KKM type theorem can be reformulated equivalently to the Fan-Browder type fixed point theorems, matching theorems, minimax inequalities, and so on.

In this section, we indicate that results of Chebbi in [7, 9] and Gourdal-Hammami in [16] can be improved following our preceding arguments.

The following is a KKM type minimax inequality given as [[32], Theorem 5.3]:

Theorem 7.1. ([32]) Let $(E, D ; \Gamma)$ be a partial KKM space. Let $f: E \times D \rightarrow \overline{\mathbb{R}}$ be an extended real-valued function and $\gamma \in \overline{\mathbb{R}}$ such that

(1) for each $y \in D,\{x \in E \mid f(x, y) \leq \gamma\}$ is intersectionally closed [resp. transfer closed];

(2) for each $N \in\langle D\rangle$ and $x \in \Gamma_{N}, \min \{f(x, y) \mid y \in N\} \leq \gamma$; and 
Suppose that there exists a nonempty compact subset $K$ of $E$ satisfying

(3) for each $N \in\langle D\rangle$, there exist $D^{\prime} \subset D$ containing $N$ and a compact $\Gamma$-convex subset $L_{N}$ of $E$ relative to $D^{\prime}$ such that

$$
L_{N} \cap \bigcap_{y \in D^{\prime}}\{x \in E \mid f(x, y) \leq \gamma\} \subset K .
$$

Then (a) there exists a $\hat{x} \in E[$ resp. $\hat{x} \in K]$ such that

$$
f(\hat{x}, y) \leq \gamma \quad \text { for all } y \in D ; \text { and }
$$

(b) if $E=D$ and $\gamma=\sup _{x \in E} f(x, x)$, then we have the minimax inequality:

$$
\inf _{y \in E} \sup _{x \in E} f(x, y) \leq \sup _{x \in E} f(x, x) .
$$

Corollary 7.1. (Chebbi [7]) Let $X$ be a nonempty convex subset of a t.v.s. E, and $f: X \times X \rightarrow \overline{\mathbb{R}}$ be a function satisfying

(i) $f$ is l.s.c. in the first variable on each compact convex subsets of $X$;

(ii) for each $A \in\langle X\rangle, \sup _{x \in \operatorname{co} A} \min _{y \in A} f(x, y) \leq 0$; and

(iii) the coercivity condition (IV) with $X=Z$ and

$$
F(y):=\{x \in X \mid f(x, y) \leq 0\} \text { for } y \in X .
$$

Then there exists an $x_{0} \in X$ such that $f\left(x_{0}, y\right) \leq 0$ for all $y \in X$.

Proof. Note that $X$ can be regarded a convex space in the sense of Lassonde [20] and endowed the compactly generated extension of of its original topology. Then (i) becomes simply ' $f$ is l.s.c.' and hence, condition (1) of Theorem 7.1 is satisfied. Moreover, it is clear that (ii) implies (2) of Theorem 7.1. Further, (iii) implies the coercivity condition (I) in Section 4 with $s=1_{E}$ and $G(y):=\{x \in E \mid f(x, y) \leq \gamma\}$ for $y \in D$. Therefore, the conclusion of Corollary 7.1 follows from Theorem 7.1(a) with $\gamma=0$.

Recall that Condition (ii) in [IV] is redundant.

Corollary 7.1 is applied to some equilibrium problems in [7] and to some quasi-variational inequalities in [9]. Note that Corollary 7.1 can be improved by adopting more general conditions [I]-[III] with $s=1_{E}$ and $Z=E$. Moreover, any interested reader can check that all results in [7] and [9] can be improved by applying Theorem 7.1 instead of Corollary 7.1.

In 2007, Gourdal-Hammami [16] applied a form of Ky Fan's matching theorem to minimax and variational inequalities. As usually some of the L-space theorists do, they listed our [38] in the references, but nothing was quoted from it.

For any subset $A$ of $\mathbb{R}$ and every $z \in \mathbb{R}, A \leq z$ denotes for all $a \in A, a \leq z$ and $A \not \leq z$ means that there exists $a \in A$ such that $a>z$.

Definition 7.1. ([16]) Let $X$ be a topological space. A correspondence $Q: X \multimap \mathbb{R}$ is said to be weakly lower semi-continuous (weakly l.s.c) on $X$ if for each $p \in \mathbb{R}$, the set $\{x \in X \mid Q(x) \leq p\}$ is closed in $X$ or equivalently, the set $\{x \in X \mid Q(x) \cap] p,+] \neq \emptyset\}$ is open in $X$.

Proposition 7.1. ([16]) If $Q$ is a lower semi-continuous correspondence then it is weakly lower semicontinuous.

The Ky Fan minimax inequality can be extended in the following way: 
Theorem 7.2. Let $(E ; \Gamma)$ be an abstract convex space and $z \in \mathbb{R}$. Let $F$ and $G$ be two correspondences from $E \times E$ to $\mathbb{R}$ satisfying the following condition:

(a) for every $x \in E, G(x, x) \leq z$,

(b) for each fixed $y \in E,\{x \in X \mid G(x, y) \leq z\}$ is $\Gamma$-convex,

(c) for each fixed $x \in E, y \mapsto F(x, y)$ is weakly l.s.c on the compact subsets of $E$,

(d) for every $(x, y) \in E \times E, F(x, y) \subset G(x, y)$,

(e) there exists a family $\left\{\left(C_{x}, K\right)\right\}_{x \in E}$ of pairs of sets satisfying:

(1) $K$ is a compact subset of $E$,

(2) for each $N \in\langle E\rangle$, there exists a compact $\Gamma$-convex set $L_{N}$ containing $N$ such that:

$$
x \in L_{N} \Rightarrow C_{x} \subset L_{N},
$$

(3) $\left\{y \in X: F(x, y) \leq z\right.$ for all $\left.x \in C_{y}\right\} \subset K$.

Then there exists $y_{0} \in X$ such that $F\left(x, y_{0}\right) \leq z \forall x \in X$.

Proof. For each $x \in X$, let $\tilde{F}(x)=\{y \in F(x): F(x, y) \leq z\}$ and $\tilde{G}(x)=\{y \in X: G(x, y) \leq z\}$. Then by (c), the correspondence $\tilde{F}$ has closed values. By (b), the set $\tilde{G}^{*}(y)=\{x \in X: G(x, y) \not \leq z\}$ is a $\Gamma$-convex subset of $X$. By (d), for each $x \in X, \tilde{G}(x) \subset \tilde{F}(x)$. Note that, by (a), for each $x \in X, x \in \tilde{G}(x)$ and $\left\{\left(C_{x}, K\right)\right\}_{x \in X}$ is a coercing family of $\tilde{F}$. Then all the requirements of Corollary 5.3 with $s$ the identity function are satisfied, hence $\bigcap_{x \in X} \tilde{F}(x) \neq \emptyset$ and the corollary is proved.

The following is [[16], Theorem 2.4]:

Corollary 7.2. ([16]) Let $(X, \Gamma)$ be an $L$-space and $z \in \mathbb{R}$. Let $F$ and $G$ be two correspondences from $X \times X$ to $\mathbb{R}$ satisfying the following condition:

(a) for every $x \in X, G(x, x) \leq z$,

(b) for each fixed $y \in X,\{x \in X \mid G(x, y) \leq z\}$ is L-convex,

(c) for each fixed $x \in X, y \mapsto F(x, y)$ is weakly l.s.c on the quasi-compact subsets of $X$,

(d) for every $(x, y) \in X \times X, F(x, y) \subset G(x, y)$,

(e) there exists a family $\left\{\left(C_{x}, K\right)\right\}_{x \in X}$ of pairs of sets satisfying:

(1) $K$ is a quasi-compact subset of $X$,

(2) for each $A \in\langle X\rangle$, there exists a quasi-compact L-convex set $D^{A}$ containing $A$ such that:

$$
x \in D^{A} \Rightarrow C_{x} \subset D^{A},
$$

(3) $\left\{y \in X: F(x, y) \leq z\right.$ for all $\left.x \in C_{y}\right\} \subset K$.

Then there exists $y_{0} \in X$ such that $F\left(x, y_{0}\right) \leq z \forall x \in X$.

\section{Historical remarks}

In this section, we give some remarks on the history of L-spaces:

(1) In 1993 [39], we introduced the generalized convex (G-convex) space $(E, D ; \Gamma)$ with the monotonicity condition. In 1998, we gave the new definition removing the condition; see [21, 25].

(2) Apparently motivated by our G-convex spaces, in 1998, the L-space $(E, \Gamma)$ was appeared in [4], where it was stated that the G-convex space $(E, \Gamma)$ in [39] with the monotonicity was particular to L-space. This false statement influenced the L-space theorists, who used to refer [39] until 2018 for twenty years; e.g. see [1] in 2018. They did not mind that G-convex spaces are triples $(E, D ; \Gamma)$ in [39. Moreover, according to the definition of L-spaces, the original KKM example [19] in 1929 and the 1961 Fan example [13] can not be L-spaces. See Section 3. 
(3) Consequently, as we have seen in Part I, there have been many mathematicians who can not distinguish pairs and triples. Some of them are concentrating only L-spaces in the KKM theory. So that such peoples can be adequately called the L-space theorists in this paper.

(4) The L-space theorists mainly work for L-spaces and their subclasses, e.g., H-spaces and topological vector spaces, as can be seen in Sections 5-7. Sometimes, they also work for triples; e.g. Corollaries 5.1, 5.2, 5.6 , 5.7, and 5.8. Consequently, they work for G-convex spaces unconsciously. They could not recognize the existence of much more broad world of abstract convex spaces for the KKM theory.

(5) The L-space theorists use inadequate concepts like quasi-compactly closed, compactly closed, strongly compactly closed, qusi-compactly open, compactly open, etc. These concepts are artificial, hard to use, not practical, and can be eliminated by adopting new topology by elementary topological method; see [22, 36].

(6) The L-space theorists introduced several coercing families. However, all of them are simple consequences of known coercivity condition (ii) in Theorem 2.1 originated from S. Y. Chang [6] in 1989.

(7) After two decades of enduring the falsehood of L-spaces, we declared that "The rise and fall of Lspaces" in Part I. Moreover, we have to also destroy the works on coercing families which are not elegant and full of artificial things. This is why we write this Part II.

From now on, we introduce some inadequate statements given by other authors:

(8) [12] "The following generalization of KKM principle obtained in [5] will be used in the proof of the main result of this paper."

Since the result in [5] was already improved in this paper, so the paper [12] needs to improve.

(9) [2] "The concept of an L-space is actually a small refinement of that of a $c$-space of Horvath. The original G-convex spaces of Park and Kim [39] are L-spaces (with an auxiliary set $D$ much as in the way of Lassonde convex spaces) where, in addition, the L-structure $\Gamma$ is isotone. Later, Park removed the isotony condition in his definition of G-convexity."

This is a false statement. The original G-convex space of Park and Kim [39] is a triple and L-space is a pair. How could a triple be a pair? Moreover Lassonde convex space [20] is a pair without $D$. The triple on convex spaces was first introduced by ourselves; see Section 3 and [25, 35]. Further, no one of the L-space theorists seems to give examples of L-spaces not satisfying the isotonicity.

(10) [1] "We note also that to define the structure of L-convexity, we do not require that $\Gamma$ satisfies a monotony type condition, i.e. if $A \subset B$, then $\Gamma(A) \subset \Gamma(B)$ as it is used by Park and Kim in [39] to define G-convex spaces and by many other papers in the literature dealing with G-convex spaces."

Since 1998, no papers on G-convex spaces by ourselves adopt the monotonicity. The authors' claim "many other papers ..." is a bluffing. It is doubtful that all of the L-space theorists quoting [39] actually read it. Because they stated false statements for two decades continuously. As we have seen in Section 3, L-spaces theory can not applicable to the KKM theorem, the $1961 \mathrm{KKMF}$ principle, and many other important results in the KKM theory; see [35. Therefore, we conclude again that L-spaces should be destroyed.

\section{References}

[1] N. Altwaijry, S. Ounaies, and S. Chebbi, Generalized convexity and applications to fixed points and equilibria, J. Fixed Point Theory Appl. (2018):3 https://doi.org/10.1007/s11784-018-0517-6

[2] H. Ben-El-Mechaiekh, Approximations and selections methods for set-valued maps and fixed point theory, Book Chapter, Research Gate, 05 Dec. 2016.

[3] H. Ben-El-Mechaiekh, S. Chebbi, M. Florenzano, and J.V. Llinares, Fixed point theorem without convexity, Working Paper 97-22 Economics Series, 11 April 1997, Departamento de Economia Universidad Carlos ill de Madrid CaIle Madrid.

[4] H. Ben-El-Mechaiekh, S. Chebbi, M. Florenzano, and J.V. Llinares, Abstract convexity and fixed points, J. Math. Anal. Appl. 222 (1998) 138-150.

[5] H. Ben-El-Mechaiekh, S. Chebbi, and M. Florenzano, A generalized KKMF principle, J. Math. Anal. Appl. 309 (2005) $583-590$. 
[6] S. Y. Chang, A generalization of KKM principle and its applications, Soochow J. Math. 15 (1989), 7-17.

[7] S. Chebbi, Minimax inequality and equilibria with a generalized coercivity, J. Appl. Anal. 12 (2006), 117-125.

[8] S. Chebbi, Intersection, fixed points and minimax inequalities with a generalized convexity in H-spaces, Arab J. Math. Sc. 12(1) (2006) 7-15.

[9] S. Chebbi, Some non-compact quasi-variational inequalities, Nonlinear Anal. 71(12) (2009) e1684-e1687.

[10] S. Chebbi, Intersection and minimax inequality with a generalized convexity in H-spaces, ResearchGate, 21 May 2015.

[11] S. Chebbi, P. Gourdel, and H. Hammami, A generalization of Fan's matching theorem, J. Fixed Point Theory Appl. 9 (2011) 117-124.

[12] S. Chebbi and B. Samet, Noncompact equilibrium points for set-valued maps, Abstract Appl. Anal. 2014, Article ID 959612, 4pp. http://dx.doi.org/10.1155/2014/959612

[13] K. Fan, A generalization of Tychonoff's fixed point theorem, Math. Ann. 142 (1961) 305-310.

[14] K. Fan, Fixed-point and related theorems for noncompact convex sets, [in: Game Theory and Related Topics (O. Moeshlin and D. Pallaschke, Eds.), pp.151-156, North-Holland, Amsterdam, 1979.

[15] K. Fan, Some properties of convex sets related to fixed point theorems, Math. Ann. 266 (1984) 519-537.

[16] P. Gourdel and H. Hammami, Applications of generalized Ky Fan's matching theorem in minimax and variational inequality, 2007. ffhalshs-00204627f

[17] H. Hammami. A generalized FKKM theorem and variational inequality, 2007. ffhalshs-00204601ff

[18] C. Horvath, Contractibility and generalized convexity, J. Math. Anal. Appl. 156 (1991) 341-357.

[19] B. Knaster, K. Kuratowski, und S. Mazurkiewicz, Ein Beweis des Fixpunktsatzes für n-Dimensionale Simplexe, Fund. Math. 14 (1929) 132-137.

[20] M. Lassonde, On the use of KKM multifunctions in fixed point theory and related topics, J. Math. Anal. Appl. 97 (1983) 151-201.

[21] S. Park, Ninety years of the Brouwer fixed point theorem, Vietnam J. Math. 27 (1999) 187-222.

[22] S. Park, Remarks on topologies of generalized convex spaces, Nonlinear Funct. Anal. Appl. 5 (2000) 67-79.

[23] S. Park, Remarks on $\mathfrak{K C}$-maps and $\mathfrak{K O}$-maps in abstract convex spaces, Nonlinear Anal. Forum 12(1) (2007) $29-40$.

[24] S. Park, Examples of $\mathfrak{K C}$-maps and $\mathfrak{K O}$-maps on abstract convex spaces, Soochow J. Math. 33(3) (2007) $477-486$.

[25] S. Park, A brief history of the KKM theory, RIMS Kôkyûroku, Kyoto Univ. 1643 (2009) 1-16.

[26] S. Park, Generalizations of the Nash equilibrium theorem in the KKM theory, Takahashi Legacy, Fixed Point Theory Appl., vol. 2010, Article ID 234706, 23pp. doi:10.1155/2010/234706.

[27] S. Park, The KKM principle in abstract convex spaces: Equivalent formulations and applications, Nonlinear Anal. 73 (2010) 1028-1042.

[28] S. Park, A genesis of general KKM theorems for abstract convex spaces, J. Nonlinear Anal. Optim. 2 (2011) $133-146$.

[29] S. Park, Remarks on certain coercivity in general KKM theorems, Nonlinear Anal. Forum 16 (2011) 1-10.

[30] S. Park, Applications of some basic theorems in the KKM theory [in: The series of papers on S. Park's Contribution to the Development of Fixed Point Theory and KKM Theory], Fixed Point Theory Appl. vol. 2011, 2011:98. DOI:10.1186/16871812-2011-98.

[31] S. Park, Evolution of the 1984 KKM theorem of Ky Fan, Fixed Point Theory Appl. vol. 2012, 2012:146. DOI:10.1186/16871812-2012-146.

[32] S. Park, On some new Ky Fan type minimax inequalities in abstract convex spaces, Nonlinear Analysis and Convex Analysis (NACA 2011, Busan), II, pp. 141-161, Yokohama Publ., Yokohama, 2012.

[33] S. Park, A genesis of general KKM theorems for abstract convex spaces: Revisited, J. Nonlinear Anal. Optim. 4(1) (2013) 127-132.

[34] S. Park, Generalizations of the KKMF principle having coercing families, J. Nonlinear Anal. Optim. 4(2) (2013) 30-40.

[35] S. Park, Comments on "Some remarks on Park's abstract convex spaces", Nonlinear Anal. Forum 20 (2015) $161-165$.

[36] S. Park, Some use of weak topologies in the KKM theory, RIMS Kôkyûroku, Kyoto Univ. 2065 (Aug. 31 - Sep. 2, 2016 ), Apr. 2018, 51-62.

[37] S. Park, On further examples of partial KKM spaces, J. Fixed Point Theory, 2019, 2019:10 (18 June, 2019$)$ 1-18.

[38] S. Park, The rise and fall of L-spaces, Adv. Th. Nonlinear Anal. Appl. 4(3) (2020) 152-166.

[39] S. Park and H. Kim, Admissible classes of multifunctions on generalized convex spaces, Proc. Coll. Natur. Sci. SNU 18 (1993) 1-21. 